\title{
Intravenous zoledronic acid for the treatment of osteoporosis: The evidence of its therapeutic effect
}

This article was published in the following Dove Press journal:

Core Evidence

16 June 2009

Number of times this article has been viewed

\section{E Michael Lewiecki}

New Mexico Clinical Research and Osteoporosis Center, Albuquerque, New Mexico, USA
Correspondence: E Michael Lewiecki Osteoporosis Director, New Mexico Clinical Research and Osteoporosis Center, 300 Oak St NE, Albuquerque, NM 87I06, USA Email lewiecki@aol.com
Introduction: Osteoporosis is a disease characterized by low bone mineral density and poor bone quality resulting in reduced bone strength and increased risk of fracture. Oral bisphosphonates, first-line therapy for most patients with osteoporosis, are associated with suboptimal adherence to therapy due to factors that include a complex dosing regimen and gastrointestinal intolerance in some patients. Intravenous bisphosphonates address these limitations through infrequent injectable dosing that assures $100 \%$ bioavailability. Intravenous zoledronic acid is the newest bisphosphonate to be approved for the treatment of osteoporosis.

Aims: This review assesses the evidence for the therapeutic effects of intravenous zoledronic acid for the treatment of osteoporosis.

Evidence review: Zoledronic acid $5 \mathrm{mg}$ administered as an annual 15-min intravenous infusion has been shown to reduce the risk of vertebral fractures, hip fractures, and other fractures in a three-year randomized, double-blind, placebo-controlled trial in women with postmenopausal osteoporosis. In a randomized, double-blind, placebo-controlled trial in women and men with a recent surgical repair of low-trauma hip fracture, it reduced the risk of new clinical fractures and improved survival. In both studies, zoledronic acid was associated with a good safety profile and was generally well tolerated. Zoledronic acid has the potential to improve clinical outcomes by reducing the risk of fracture in patients with osteoporosis.

Clinical value: Intravenous zoledronic acid $5 \mathrm{mg}$ every 12 months reduces fracture risk in women with postmenopausal osteoporosis and in women and men with recent low-trauma hip fracture.

Keywords: osteoporosis, zoledronic acid, treatment, management, fracture, bisphosphonates

Core evidence clinical impact summary for once yearly intravenous zoledronic acid in the treatment of osteoporosis

\begin{tabular}{|c|c|c|}
\hline Outcome measure & Evidence & Implications \\
\hline \multicolumn{3}{|l|}{ Patient-oriented evidence } \\
\hline Fracture risk reduction & Clear & $\begin{array}{l}\text { Reduced risk of vertebral, hip, and nonvertebral } \\
\text { fractures in women with postmenopausal } \\
\text { osteoporosis and reduced risk of clinical fractures in } \\
\text { men and women with recent low-trauma hip fracture }\end{array}$ \\
\hline Reduced mortality & Moderate & $\begin{array}{l}\text { All-cause mortality rate may be decreased in patients } \\
\text { treated after recent low-trauma hip fracture }\end{array}$ \\
\hline \multicolumn{3}{|l|}{ Disease-oriented evidence } \\
\hline Bone density increase & Clear & Increase in bone density at lumbar spine and hip \\
\hline Bone turnover suppression & Clear & $\begin{array}{l}\text { Rapid suppression of bone turnover markers that is } \\
\text { sustained for at least one year }\end{array}$ \\
\hline \multicolumn{3}{|l|}{ Economic evidence } \\
\hline Cost-effectiveness & Moderate & $\begin{array}{l}\text { Higher cost of zoledronic acid compared with generic } \\
\text { oral bisphosphonate may, in part, be offset by assured } \\
\text { therapeutic effect for at least one year after infusion }\end{array}$ \\
\hline
\end{tabular}




\section{Scope, aims, and objectives}

Zoledronic acid (zoledronate) $5 \mathrm{mg}$ (Reclast $^{\circledR}$ in the US, Aclasta $^{\circledR}$ in other countries; Novartis Pharmaceutical Corporation, East Hanover, NJ, USA) is approved by the FDA as a once yearly treatment for osteoporosis in postmenopausal women, diagnosed by bone mineral density (BMD) or prevalent vertebral fracture, and for treatment of patients (men and women) at high risk of fracture, defined as a recent low-trauma hip fracture. It is approved as a singledose treatment of Paget's disease of bone in men and women. Zoledronic acid is also available as a $4 \mathrm{mg}$ dose (Zometa $^{\circledR}$, Novartis Pharmaceutical Corporation, East Hanover, NJ, USA) that is FDA-approved for the treatment of hypercalcemia of malignancy, multiple myeloma, and bone metastases of solid tumors, given as often as every 3-4 weeks.

Zoledronic acid is an aminobisphosphonate with a high affinity for mineralized bone. Administered as an intravenous infusion over at least $15 \mathrm{~min}$, it rapidly localizes to bone, where it inhibits osteoclastic bone resorption by inhibiting the action of the enzyme farnesyl pyrophosphate synthase in the mevalonate pathway. The relatively long duration of action of zoledronic acid is attributable to its high binding affinity for bone mineral.

This review addresses the evidence for the use of zoledronic acid in the treatment of osteoporosis in women and men. Zoledronic acid for the treatment of Paget's disease of bone and cancer-related conditions is not considered here.

\section{Methods}

Evidence for the role of zoledronic acid in the treatment of osteoporosis was obtained through a literature search conducted on August 25, 2008, using the terms "zoledronic acid" and "osteoporosis" (search 1) or "fracture" (search 2) in the PubMed (http://www.ncbi.nlm.nih.gov/pubmed/) database with limits of "humans," "clinical trials," and "English." Search 1 identified 26 matches and search 2 identified 46 matches. A review of all matches eliminated duplicates, review articles, studies in patients with cancer-related conditions, and studies not having fractures as the primary endpoint. A search of the National Guideline Clearinghouse (http://www.guide.gov), the Cochrane Database of Systematic Reviews (http://cochrane.org/index0. htm), and Evidence Based Medicine Reviews (http://www.acpjc. org) using the same search terms revealed no additional matches meeting study criteria. Two large phase III randomized, doubleblind, placebo-controlled clinical trials with fractures as a primary endpoint were found: Health Outcomes and Reduced Incidence with Zoledronic Acid Once Yearly (HORIZON)
Pivotal Fracture Trial (PFT) ${ }^{1}$ and HORIZON Recurrent Fracture Trial (RFT). ${ }^{2}$ Each study provided level 2 evidence on the efficacy and safety of zoledronic acid for the treatment of osteoporosis. A third study of clinical relevance, also with level 2 evidence but not powered to assess differences in fracture risk, was evaluated. ${ }^{3}$ This was a randomized, doubleblind, double-dummy, multicenter trial assessing the safety and efficacy of a single dose of zoledronic acid $5 \mathrm{mg}$ versus alendronate $70 \mathrm{mg} /$ week in postmenopausal women previously treated with alendronate. These studies provided the focus of this evidence review, with other supporting studies presented when appropriate to enhance understanding of the use of zoledronic acid in the treatment of osteoporosis.

Table 1 summarizes the levels of evidence of articles identified from the search strategy.

\section{Disease overview}

Osteoporosis is a disease characterized by low BMD and poor bone quality that results in reduced skeletal strength and increased risk of fracture. ${ }^{4}$ It is diagnosed by measuring a patient's BMD by dual-energy x-ray absorptiometry (DXA) and applying criteria established by the World Health Organization (WHO). ${ }^{5}$ If a fragility fracture has occurred, a clinical diagnosis of osteoporosis may be made independently of BMD once other causes of skeletal fragility have been eliminated. There are no symptoms of osteoporosis until a fracture occurs.

Osteoporosis and low bone mass (osteopenia) are common conditions that affect about 44 million people in the US. ${ }^{6}$ Worldwide, osteoporosis is estimated to be present in over 200 million individuals. ${ }^{7}$ While osteoporosis can occur at

Table I Evidence base included in the review

\begin{tabular}{lll}
\hline Category & \multicolumn{2}{l}{ Number of records } \\
\cline { 2 - 3 } & Full papers & Abstracts \\
\hline Initial search & 72 & 0 \\
Records excluded & 70 & 0 \\
Records included & 2 & 0 \\
Additional studies identified & 1 & 0 \\
Level I clinical evidence & 0 & 0 \\
Level 2 clinical evidence & 3 & 0 \\
Level $\geq 3$ clinical evidence & 0 & 0 \\
Trials other than RCT & 0 & 0 \\
Case reports & 0 & 0 \\
Economic evidence & 0 & 0
\end{tabular}

Notes: For definitions of levels of evidence see Core Evidence website (http://www. dovepress.com/core-evidence-journal).

Abbreviation: RCT, randomized controlled trial. 
any age, it becomes more common with aging, predisposing many older individuals to fractures. ${ }^{4}$ About $55 \%$ of adults aged 50 and older are at risk, $80 \%$ of whom are women. ${ }^{6}$ An estimated $50 \%$ of women and $25 \%$ of men above age 50 will sustain an osteoporotic fracture of the hip, spine, or wrist during the remainder of their lifetime. ${ }^{6}$ Fracture risk increases with decreasing BMD, advancing age, and in the presence of clinical risk factors that include previous fracture, parent with hip fracture, current cigarette smoking, chronic glucocorticoid use, rheumatoid arthritis, and excess alcohol intake. ${ }^{8}$ The burden of osteoporosis is expected to worsen in the future, in part due to the aging of the population. In 2005 there were an estimated two million new osteoporotic fractures in the US; by 2025 , that number is predicted to increase to three million. ${ }^{9}$

Osteoporotic fractures are associated with substantial morbidity and mortality. These fractures may cause acute and chronic pain, diminished quality of life, and high healthcare expenses for the individual and society. Nearly one-third of hip fracture patients enter a nursing home within a year of their fracture, and $20 \%$ of osteoporotic hip fracture patients die within that same time period. ${ }^{4}$ Loss of independence is a major fear of the elderly, with $80 \%$ of women above the age of 75 preferring death to placement in a nursing home as a result of a hip fracture. ${ }^{4}$ Fractures of the hip, spine, or wrist have been found to affect quality of life measures to a degree that is similar to that seen in other serious chronic diseases such as asthma, chronic obstructive pulmonary disease, and osteoarthritis. $^{10}$

The total direct cost of osteoporosis-related medical care is estimated to be \$US17 billion annually in the US ${ }^{9}$ and $€ 31.7$ billion in the EU. ${ }^{8}$ Hip fractures account for $14 \%$ of incident fractures and $72 \%$ of fracture costs, with the number of hip fractures and associated costs expected to double or triple by $2040 .{ }^{11}$ Following a fracture, a patient's overall healthcare expenses increase as well. In a population-based study of patients from Olmsted County, Minnesota, US, the direct medical costs in the year following a fracture increased from \$US761 to \$US3884 per patient. ${ }^{12}$ Indirect costs due to lost wages and diminished productivity of the patient and/or caregiver were not considered in this analysis. It is likely that the true socioeconomic burden of osteoporotic fractures is greatly underestimated by most studies. ${ }^{4}$

In collaboration with numerous medical societies, the National Osteoporosis Foundation (NOF) developed and recently released an updated comprehensive clinicians' guide for the management of osteoporosis. ${ }^{13}$ This guide is intended to be used by clinicians to assist in making decisions in the evaluation and treatment of individual patients. It provides indications for BMD testing in postmenopausal women and men aged 50 years and older, and suggests ways to evaluate patients for factors contributing to low BMD and skeletal fragility. NOF treatment recommendations are based on BMD and/or personal history of fracture, or an assessment of 10-year fracture probability according to a US adaptation of the WHO fracture risk algorithm, FRAX ${ }^{\mathrm{TM}} .{ }^{14}$ The NOF guide focuses on providing clinical tools for the identification of patients who are at high risk of fracture and most likely to benefit from pharmacologic intervention.

\section{Current therapeutic options}

Universal recommendations for skeletal health in all adults over age 50 include a daily intake of at least $1200 \mathrm{mg}$ elemental calcium with diet, plus supplements, if needed, and vitamin $\mathrm{D}_{3}$ 800-1000 IU. ${ }^{13}$ Regular weight-bearing exercise and muscle-strengthening exercise to reduce the risk of falls and fractures is advised. Cigarette smoking and excess alcohol intake (three or more drinks per day) should be avoided.

Medications approved for osteoporosis prevention include estrogen, raloxifene (an estrogen receptor agonist/ antagonist), and three bisphosphonates, alendronate, risedronate, and ibandronate, all of which are classified as antiresorptive (anticatabolic) agents that inhibit osteoclastic bone resorption. Those approved for osteoporosis treatment include antiresorptive drugs raloxifene, alendronate, risedronate, ibandronate, zoledronic acid, and salmon calcitonin, and one anabolic (bone-forming) drug, teriparatide [parathyroid hormone (PTH) 1-34]. ${ }^{13}$ Other drugs, such as strontium ranelate, etidronate, and PTH (1-84) are approved in some countries but not the US.

Registration of a drug for the treatment of osteoporosis in the US and the EU requires demonstration of vertebral fracture risk reduction in a phase III randomized, double-blind, placebo-controlled clinical trial. Some approved drugs have been proven to reduce fractures at other skeletal sites as well. For example, there is evidence that alendronate, risedronate, and zoledronic acid reduce hip fracture and nonvertebral fracture risk, ${ }^{1,15-18}$ and teriparatide has been shown to reduce nonvertebral fracture risk. ${ }^{19}$ Estrogen has proven fracture risk reduction at the spine, hip, and other skeletal sties, but is not recommended as first-line therapy for the treatment of osteoporosis due to adverse events associated with longterm use. All of these drugs are approved for the treatment of postmenopausal osteoporosis, while alendronate, risedronate, 
zoledronic acid, and teriparatide are currently approved for treatment of osteoporosis in men.

The registration trials for alendronate, risedronate, and ibandronate were conducted with daily dosing of medication, although the dosing interval most often used now is weekly for alendronate, weekly or monthly for risedronate, and monthly for oral ibandronate. Ibandronate may also be given as an intravenous infusion every three months. Raloxifene, nasal calcitonin, teriparatide, and strontium ranelate require daily administration. The dosing regimen for oral bisphosphonates requires a 30-min postdose fast for alendronate and risedronate, and a 60 -min postdose fast for ibandronate. Oral bisphosphonates have been associated with gastrointestinal intolerance in some clinical practice patients, although in clinical trials there have been no significant differences in gastrointestinal adverse events in bisphosphonate-treated patients versus those on placebo. Rarely, bisphosphonates have been associated with a syndrome of persistent myalgias and arthralgias that may recur on rechallenge. ${ }^{20}$ Bisphosphonates, particularly when given intravenously for cancer patients, such as those with multiple myeloma or breast carcinoma, have been associated with osteonecrosis of the jaw. ${ }^{21}$ Raloxifene and estrogen share an increased incidence of venous thromboembolic events. ${ }^{22,23}$ Raloxifene is also associated with an increased incidence of leg cramps and vasomotor symptoms ${ }^{24}$ while estrogen reduces vasomotor symptoms. ${ }^{18}$ Teriparatide has been linked with osteosarcoma in rodent models. ${ }^{25}$ Osteosarcoma did not occur when the rodents had exposure of less than $70 \%$ of their lifespan, ${ }^{26}$ and does not appear to be a problem in adult humans treated with approved doses for osteoporosis. ${ }^{27}$ Teriparatide is also associated with transient hypercalcemia 4-6 hours after injection and elevation of uric acid not associated with clinical gout. ${ }^{19}$

\section{Unmet needs}

Despite the availability of excellent diagnostic tools and evidence demonstrating the efficacy of medications to treat osteoporosis and reduce fracture risk, a significant proportion of patients with osteoporosis is undiagnosed and untreated. ${ }^{11,28}$ Even when patients are treated for osteoporosis, it is common for medication to be taken incorrectly or for too short a time to provide benefit in terms of fracture risk reduction. $^{29}$

The multinational IMPACT study ${ }^{30}$ evaluated the accuracy of radiographic diagnoses of vertebral fracture in postmenopausal women with a new diagnosis of osteoporosis based on BMD measurements. Of 2451 women with evaluable lateral spine radiographs, $789(32 \%)$ were found to have at least one vertebral fracture, and 266 (34\% of those with fractures) were not recognized as having a vertebral fracture in reports by local radiologists. In a study that analyzed hospital records for patients diagnosed with osteoporosis and/or osteoporotic fractures, the annual incidence of osteoporosis in women 50 years or older was estimated to be 58568 per million inhabitants; however, only 4823 cases were diagnosed, representing just $8.2 \%$ of the expected figure. ${ }^{31}$ In another study of 95 patients aged 65 years or older having surgery for hip fracture, none had a BMD assessment during hospitalization or were scheduled to have it as an outpatient procedure, and only $2 \%$ reported receiving pharmacologic therapy for osteoporosis at discharge. ${ }^{32}$

Adherence to treatment is less than optimal. Nearly $50 \%$ of all patients started on weekly alendronate or risedronate remain on treatment for less than one year. ${ }^{33}$ A study of a large US pharmacy claims database showed that one-year adherence was better with weekly dosing compared with daily (45\% compared with $33 \%$, respectively), but was still poor. ${ }^{34}$ Many patients express a preference for monthly compared with weekly dosing of an oral bisphosphonate. ${ }^{35}$ Persistence with a monthly drug treatment regimen may be better than with weekly, although is still suboptimal. ${ }^{36}$ Poor adherence to therapy is associated with adverse clinical outcomes, including greater risk of fractures ${ }^{29}$ and higher healthcare costs. ${ }^{36}$

\section{Clinical evidence with zoledronic acid for the treatment of osteoporosis}

The key patient-oriented outcome of fractures with zoledronic acid therapy for the treatment of osteoporosis has been evaluated in two randomized controlled trials (RCTs): HORIZONPFT $^{1}$ and HORIZON-RFT. ${ }^{2}$ Both studies assessed the disease-oriented outcome of BMD, and HORIZON-PFT, but not HORIZON-RFT, measured bone turnover markers.

HORIZON-PFT was a three-year international, multicenter, randomized, double-blind, placebo-controlled trial that enrolled 7765 postmenopausal women (age 65-89 years, mean 73 years) with osteoporosis, defined as a BMD T-score of -2.5 or less at the femoral neck with or without evidence of existing vertebral fracture, or a T-score of -1.5 or less with radiologic evidence of at least two mild or one moderate vertebral fracture. Previous use of oral bisphosphonates was allowed, provided criteria for a predefined washout period were met. Concomitant use of the following osteoporosis medications was allowed at the time of entry into the study and during follow-up: hormone therapy, raloxifene, calcitonin, tibolone, tamoxifen, dehydroepiandrosterone, ipriflavone, and medroxyprogesterone. Patients were placed into 
one of two strata according to whether they were taking osteoporosis medications at baseline. Those assigned to stratum 1 were not taking any osteoporosis medications at the time of randomization, while those in stratum 2 were all taking an allowed medication. Patients with previous bisphosphonate treatment who met washout criteria were included in the study. Patients were excluded from participation for any previous use of PTH or sodium fluoride, use of anabolic steroids or growth hormone within six months of trial entry, use of oral or intravenous systemic corticosteroids within 12 months, and any previous use of strontium ranelate. Patients with a serum calcium level of more than $11 \mathrm{mg} / \mathrm{dL}(2.75 \mathrm{mmol} / \mathrm{L})$ or less than $8 \mathrm{mg} / \mathrm{dL}$ ( $2 \mathrm{mmol} / \mathrm{L})$, a calculated creatinine clearance of less than $30 \mathrm{~mL} / \mathrm{min}$ at either of two baseline visits, or urine dipstick protein of more than $2+$ without evidence of contamination or bacteriuria were also excluded. Patients were randomized to receive a 15-min intravenous infusion of zoledronic acid $5 \mathrm{mg}(\mathrm{n}=3889)$ or placebo $(\mathrm{n}=3876)$ at baseline, 12 months, and 24 months. A treatment window of 90 days ( -1 month/+2 months from one-year anniversary of prior infusion) was allowed for the administration of repeat infusions. All patients received supplemental calcium 1000-1500 mg and vitamin D 400-1200 IU per day. Primary endpoints were new vertebral fractures in stratum 1 and hip fracture in both strata. Secondary efficacy endpoints included any nonvertebral fracture, any clinical fracture, and clinical vertebral fracture. Other secondary endpoints were changes in BMD at the total hip, femoral neck, and lumbar spine, and changes in bone resorption markers (serum C-telopeptide of type I collagen) and bone formation markers (bone-specific alkaline phosphatase and $\mathrm{N}$-terminal propeptide of type I collagen).

HORIZON-RFT was a randomized, double-blind, placebo-controlled trial that enrolled 2127 women and men age 50 and older (mean age, 74.5 years) with a recent low-trauma hip fracture. All enrolled patients had both legs and were walking before the hip fracture. Concomitant therapy with nasal calcitonin, selective estrogen receptor modulators, hormone replacement, tibolone, and external hip protectors was allowed. Prior use of bisphosphonates or PTH was allowed according to a prespecified washout period. Exclusions included prior use of strontium ranelate or sodium fluoride, hypersensitivity to a bisphosphonate, potential for pregnancy, calculated creatinine clearance less than $30 \mathrm{~mL} / \mathrm{min}$, corrected serum calcium level of more than $11 \mathrm{mg} / \mathrm{dL}(2.75 \mathrm{mmol} / \mathrm{L})$ or less than $8 \mathrm{mg} / \mathrm{dL}(2 \mathrm{mmol} / \mathrm{L})$, active cancer, metabolic bone disease other than osteoporosis, and a life expectancy of less than six months. The duration of the study was event driven, with 211 clinical fractures required to have a power of $90 \%$ to detect a $35 \%$ reduction in the rate of clinical fracture in the zoledronic acid group compared with the placebo group. Patients were assigned to receive a yearly 15-min intravenous infusion of zoledronic acid $5 \mathrm{mg}$ $(n=1065)$ or placebo $(n=1062)$, with the first dose administered within 90 days after surgical repair of a hip fracture. If the baseline serum 25-hydroxyvitamin D level was $15 \mathrm{ng} / \mathrm{mL}$ or less, or if the level was not available, patients received a loading dose of either vitamin $\mathrm{D}_{3}$ or $\mathrm{D}_{2}$ (at 50000-125000 IU, orally or intramuscularly) 14 days before the first infusion of a study drug. Thereafter, all patients received daily supplementation with oral calcium (1000-1500 mg) and vitamin D (800-1200 IU). The median follow-up was 1.9 years. The primary endpoint was a new clinical fracture, excluding fractures of the face and digits, and fractures in abnormal bone, such as those seen with metastatic cancer. Secondary endpoints included change in BMD in the nonfractured hip, new vertebral and nonvertebral fractures, hip fractures, and prespecified safety endpoints that included death.

In a one-year study of 225 postmenopausal women with low BMD who were previously treated with alendronate for at least one year (mean, four years), the safety and efficacy of a single intravenous dose of zoledronic acid $5 \mathrm{mg}$ versus alendronate $70 \mathrm{mg} /$ week was compared. ${ }^{3}$ All patients were between the ages of 45 and 79 years (mean age, 68 years) with a documented T-score less than or equal to -2.0 at the lumbar spine or femoral neck prior to initiation of alendronate. Patients were excluded for a calculated creatinine clearance less than $30 \mathrm{~mL} / \mathrm{min}$, or urine dipstick with more than $2+$ protein without evidence of contamination or bacteriuria; serum calcium greater than $11 \mathrm{mg} / \mathrm{dL}(2.75 \mathrm{mmol} / \mathrm{L})$ or less than $8 \mathrm{mg} / \mathrm{dL}$ ( $2 \mathrm{mmol} / \mathrm{L})$; serum alkaline phosphatase more than 1.5 times the upper limit of normal; abnormalities of the esophagus that may delay esophageal emptying; treatment with intravenous bisphosphonates within the last two years; treatment with raloxifene, calcitonin, tibolone, or hormone replacement therapy within six months prior to randomization; and any treatment with strontium ranelate, sodium fluoride, or PTH. The primary objective was to evaluate the noninferiority of intravenous zoledronic acid $5 \mathrm{mg}$ compared with weekly oral alendronate $70 \mathrm{mg}$ in maintaining BMD at the lumbar spine. Secondary objectives included comparison of bone turnover markers over 12 months.

\section{Fractures}

HORIZON-PFT ${ }^{1}$ showed that in women with postmenopausal osteoporosis, intravenous infusion of zoledronic acid 
$5 \mathrm{mg}$ every 12 months over three years significantly reduced the relative risk of morphometric vertebral fracture compared with placebo by $70 \%$ [absolute risk reduction (ARR), 7.6\%]; reduced hip fracture risk by $41 \%$ (ARR, $1.1 \%$ ); reduced clinical vertebral fracture risk by $77 \%$ (ARR, 2.1\%); reduced nonvertebral fracture risk by $25 \%$ (ARR, 2.7\%); and reduced the risk of all clinical fractures by $33 \%$ (ARR, 4.4\%) (Table 2).

In HORIZON-RFT, ${ }^{2}$ men and women treated with intravenous zoledronic acid $5 \mathrm{mg}$ within 90 days of a lowtrauma hip fracture had a significantly reduced risk of clinical fracture (35\%; ARR, 5.3\%), clinical vertebral fracture (46\%; ARR, 2.1\%), and nonvertebral fracture (27\%; ARR, 3.1\%) compared with placebo (Table 3).

\section{BMD}

BMD is a surrogate biomarker for bone strength, with stability or an increase in BMD associated with a reduction in fracture risk in patients treated with effective osteoporosis medications. ${ }^{38}$ In HORIZON-PFT, ${ }^{1}$ BMD was increased significantly at the lumbar spine (6.71\%), total hip (6.02\%), and femoral neck $(5.06 \%)$ in the zoledronic acid group versus the placebo group over three years $(P<0.001$ for all comparisons). In HORIZON-RFT, ${ }^{2}$ significant BMD increases were also observed. BMD at the total hip increased by $2.6 \%, 4.7 \%$, and $5.5 \%$ at 12,24 , and 36 months, respectively, over 3 years in the zoledronic acid group, compared with decreases of $1 \%, 0.7 \%$, and $0.9 \%$ at the corresponding time points in the placebo group. Femoral neck BMD increased in the zoledronic acid group by $0.8 \%, 2.2 \%$, and $3.6 \%$ at 12,24 , and 36 months, respectively, compared with

Table 2 Relative risk of fracture in postmenopausal women with osteoporosis with zoledronic and versus placebo in HORIZON-PFT'

\begin{tabular}{|c|c|c|c|c|}
\hline \multirow[t]{2}{*}{$\begin{array}{l}\text { Type of } \\
\text { fracture }\end{array}$} & \multicolumn{2}{|c|}{$\begin{array}{l}\text { Number of patients } \\
\text { with fractures/total } \\
\text { number of patients (\%) }\end{array}$} & \multirow[t]{2}{*}{$\begin{array}{l}\text { Relative risk } \\
\text { or hazard ratio } \\
(95 \% \mathrm{Cl})\end{array}$} & \multirow[t]{2}{*}{$P$ value } \\
\hline & Placebo & $\begin{array}{l}\text { Zoledronic } \\
\text { acid }\end{array}$ & & \\
\hline $\begin{array}{l}\text { Morphometric } \\
\text { vertebral }\end{array}$ & $\begin{array}{l}310 / 2853 \\
(10.9 \%)\end{array}$ & $\begin{array}{l}92 / 2822 \\
(3.3 \%)\end{array}$ & $0.30(0.24-038)$ & $<0.001$ \\
\hline Hip & $\begin{array}{l}88 / 3861 \\
(2.5 \%)\end{array}$ & $\begin{array}{l}52 / 3875 \\
(1.4 \%)\end{array}$ & $0.59(0.42-0.83)$ & 0.002 \\
\hline $\begin{array}{l}\text { Clinical } \\
\text { vertebral }\end{array}$ & $\begin{array}{l}84 / 3861 \\
(2.6 \%)\end{array}$ & $\begin{array}{l}19 / 3875 \\
(0.5 \%)\end{array}$ & $0.23(0.14-0.37)$ & $<0.001$ \\
\hline Nonvertebral & $\begin{array}{l}388 / 3861 \\
(10.7 \%)\end{array}$ & $\begin{array}{l}292 / 3875 \\
(8.0 \%)\end{array}$ & $0.75(0.64-0.87)$ & $<0.001$ \\
\hline
\end{tabular}

Abbreviation: $\mathrm{Cl}$, confidence interval.
Table 3 Relative risk of fracture in men and women with recent low-trauma hip fracture with zoledronic acid versus placebo in HORIZON-RFT ${ }^{2}$

\begin{tabular}{lllll}
\hline $\begin{array}{l}\text { Type of } \\
\text { fracture }\end{array}$ & $\begin{array}{l}\text { No. of patients with } \\
\text { fractures/total no } \\
\text { of patients }\end{array}$ & $\begin{array}{l}\text { Hazard } \\
\text { ratio } \\
\text { (95\% CI) }\end{array}$ & P value \\
\cline { 2 - 3 } & Placebo & $\begin{array}{l}\text { Zoledronic } \\
\text { acid }\end{array}$ & & \\
\hline All clinical & $139 / 1062$ & $92 / 1065$ & 0.65 & 0.001 \\
fractures & $(13.9 \%)$ & $(8.6 \%)$ & $(0.50-0.84)$ & \\
Nonvertebral & $107 / 1062$ & $79 / 1065$ & 0.73 & 0.03 \\
Hip & $(10.7 \%)$ & $(7.6 \%)$ & $(0.55-0.98)$ & \\
& $33 / 1062$ & $23 / 1065$ & 0.70 & 0.18 \\
Clinical & $(3.5 \%)$ & $(2.0 \%)$ & $(0.41-1.19)$ & \\
vertebral & $39 / 1062$ & $21 / 1065$ & 0.54 & 0.02 \\
\hline
\end{tabular}

Abbreviation: $\mathrm{Cl}$, confidence interval.

declines of $1.7 \%, 2.1 \%$, and $0.7 \%$ in the placebo group at corresponding time points (Figure 1). All increases in total hip and femoral neck BMD were statistically significant versus placebo $(P<0.001)$.

In postmenopausal women with low BMD who were previously treated with alendronate for at least one year, ${ }^{3}$ there was no statistical difference between the BMD response at the lumbar spine with a single dose of intravenous zoledronic acid $5 \mathrm{mg}$ versus alendronate $70 \mathrm{mg} /$ week, meeting the predefined criteria for noninferiority of zoledronic acid in the study population. The observed lumbar spine BMD changes were small in both groups, with a change from baseline to month 12 of $0.167 \%$ (SE, 0.258 ) in the group receiving zoledronic acid $5 \mathrm{mg}$ compared with $0.813 \%$ (SE, 0.283) in the alendronate $70 \mathrm{mg}$ group in the intent-to-treat population.

\section{Bone turnover markers}

Bone turnover markers are biochemical byproducts of bone remodeling that can be measured in blood and urine.

Bisphosphonates, including zoledronic acid, exert their skeletal effects through inhibition of osteoclastic bone resorption, which is reflected by a decrease in bone resorption markers. Due to coupling of bone resorption and formation, bone formation markers typically also decrease, but this occurs later than the decrease in bone resorption markers, and to a lesser magnitude.

In HORIZON-PFT, ${ }^{1}$ all three bone turnover markers decreased significantly in patients in the zoledronic acid group compared with placebo. At 12 months, levels of serum C-telopeptide of type I collagen, bone-specific alkaline phosphatase, and N-terminal propeptide of type I collagen 


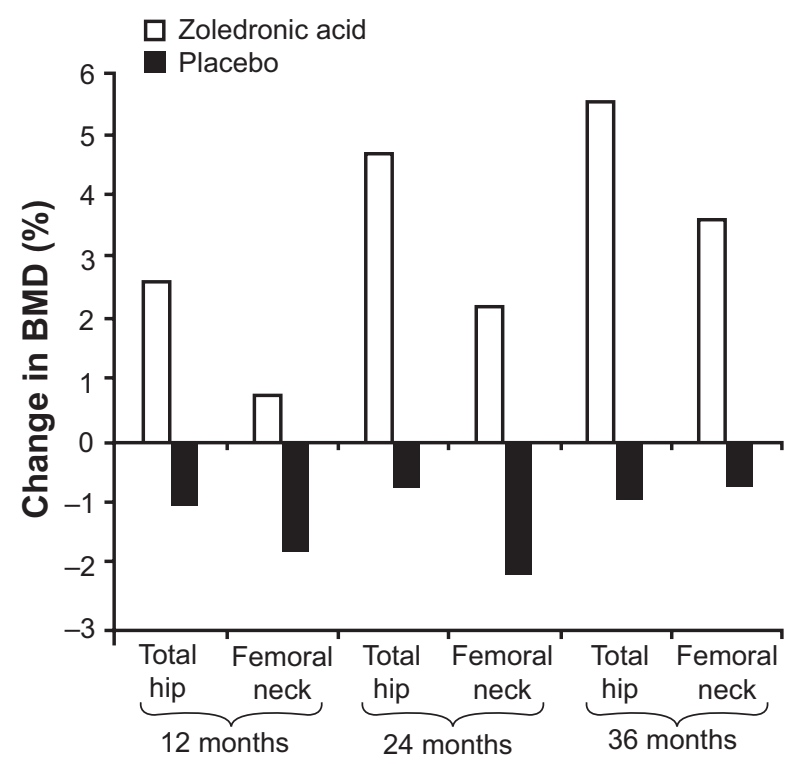

Figure I Change in BMD during 3 years' treatment with yearly zoledronic acid $5 \mathrm{mg}$ $(n=1065)$ or placebo $(n=1062)$ in patients aged $\geq 50$ years (mean age, 74.5 years) with a recent low-trauma hip fracture $(\text { HORIZON-RFT) })^{2} P<0.00$ I zoledronic acid vs placebo at all time points.

Abbreviation: BMD, bone mineral density.

were $59 \%$ (95\% confidence interval [CI]: 55, 63), 30\% (95\% CI: 27, 32), and 58\% (95\% CI: 55, 60) lower, respectively, in the zoledronic acid group $(P<0.001$ for all) compared with placebo. At 6 and 12 months after each of the three infusions, the mean values were similar, with no progressive decline in bone marker levels.

In postmenopausal women with low BMD who were previously treated with alendronate, ${ }^{3}$ a single dose of intravenous zoledronic acid $5 \mathrm{mg}$ significantly decreased bone turnover markers at three months compared with baseline, with a subsequent rise in levels to greater than baseline, yet well within the premenopausal reference range, at nine and 12 months. The decrease in bone turnover marker levels at three months with zoledronic acid suggests that alendronate does not maximally suppress bone turnover, and the subsequent rise in levels appears to represent reversibility of the potent antiresorptive effect of zoledronic acid.

\section{Safety and tolerability}

Once yearly intravenous infusion of zoledronic acid $5 \mathrm{mg}$ for the treatment of osteoporosis is associated with a favorable safety profile and is generally well tolerated. Key safety issues are discussed below.

\section{Acute phase (postdose) reaction}

The five most common postdose symptoms (within three days of infusion) reported in HORIZON-PFT ${ }^{1}$ were pyrexia
(16.1\% versus $2.1 \%$ with placebo), myalgia (9.5\% versus $1.7 \%)$, influenza-like symptoms $(7.8 \%$ versus $1.6 \%)$, headache (7.1 \% versus $2.3 \%$ ), and arthralgia (6.3\% versus $2.0 \%)$ ( $P<0.001$ for all comparisons). These symptoms typically resolved within three days of onset and declined markedly after the first infusion. Any of these postdose symptoms occurred in $31.6 \%$ of patients receiving zoledronic acid after the first infusion, 6.6\% after the second infusion, and 2.8\% after the third infusion. Administration of antipyretics (eg, acetaminophen, ibuprofen) seemed to mitigate these symptoms. In HORIZON-RFT, ${ }^{2}$ fewer acute phase reactions were seen, possibly due to the routine use of acetaminophen for up to 72 hours following the infusion.

Previous treatment with a bisphosphonate appears to provide some protection from acute phase reactions with zoledronic acid. ${ }^{3}$ Similar postdose symptoms have been observed with other bisphosphonates, administered intravenously and orally, and appear to be due, at least in part, to the release of inflammatory cytokines from circulating T cells. ${ }^{39}$

\section{Osteonecrosis of the jaw}

Osteonecrosis of the jaw (ONJ) is a rare disorder of the oral cavity that has recently been defined as the presence of exposed bone in the maxillofacial region that does not heal within eight weeks after identification by a healthcare provider. ${ }^{21}$ Most cases of bisphosphonate-related ONJ (about 94\%) have been reported in patients who receive monthly intravenous bisphosphonates for the treatment of malignancies, particularly multiple myeloma and metastatic cancer. ${ }^{40}$ Potential risk factors for ONJ include long duration of exposure to bisphosphonate treatment, glucocorticoid use, recent dental extraction, invasive oral bone surgery, poorly fitting dental appliances and/or intraoral trauma, preexisting dental or periodontal disease, cancer, anticancer therapy, and alcohol/ tobacco abuse. ${ }^{21}$

In HORIZON-PFTand HORIZON-RFT there were no spontaneous reports of ONJ. ${ }^{1,2}$ To identify potential unreported cases among HORIZON-PFT patients, the adverse event database was searched using 60 safety terms chosen by a panel of maxillofacial experts. An independent adjudication committee that was blinded to study treatment then reviewed all identified events. Possible ONJ cases were defined as exposed bone in the maxillofacial area with delayed healing of more than six weeks despite appropriate care. Two potential cases of ONJ were identified in HORIZON-PFT, one in the placebo group and one in the zoledronic acid group, with each of these resolving with antibiotic treatment and debridement. 
These findings are consistent with previous reports suggesting that the risk of ONJ in bisphosphonate-treated osteoporosis patients is low (an estimated prevalence of less than one in 100000 patient years in noncancer patients), and that the benefit of bisphosphonate therapy in preventing fractures generally far outweighs the risk of ONJ. ${ }^{21}$ A review of the adjudicated cases of ONJ in HORIZON-PFT concluded that there is no evidence to suggest that healthy patients receiving bisphosphonates for osteoporosis require any special dental treatment beyond routine care and standard procedures ${ }^{41}$ It is probably prudent for clinicians to do a routine oral examination before prescribing a bisphosphonate and to consider appropriate preventive dental care prior to treatment in patients with a history of ONJ risk factors.

\section{Renal effects}

Intravenous bisphosphonates have been associated with adverse renal effects that are primarily related to dose and infusion time, with the risk increasing with higher dose and faster infusion time. ${ }^{42}$ In HORIZON-PFT and HORIZON-RFT, using zoledronic acid $5 \mathrm{mg}$ with an infusion time of $15 \mathrm{~min}$, no long-term effects on renal function were observed. In HORIZON-PFT, ${ }^{1}$ there was a transient increase in serum creatinine (defined as a rise of more than $0.5 \mathrm{mg} / \mathrm{dL}$ ) in $1.2 \%$ of zoledronic acid patients compared with $0.4 \%$ of placebo patients $(P<0.001)$. Within 30 days after infusion, the levels returned to within $0.5 \mathrm{mg} / \mathrm{dL}$ of preinfusion values in over $85 \%$ of patients, with the remainder of patients back to this level by the time of the next annual follow up. In HORIZON-RFT, ${ }^{2}$ the incidence of serum creatinine elevations $>0.5 \mathrm{mg} / \mathrm{dL}$ did not differ between the zoledronic acid and placebo groups (6.2\% and $5.6 \%$, respectively, $P=$ not significant).

\section{Hypocalcemia}

In HORIZON-PFT, at nine to 11 days after the first infusion, $0.2 \%$ of patients in the zoledronic acid group had a decrease in serum calcium level to less than $7.5 \mathrm{mg} / \mathrm{dL}^{1}$ as $\operatorname{did} 0.3 \%$ of those in HORIZON-RFT zoledronic acid group. ${ }^{2}$ All events were transient and asymptomatic.

\section{Ocular events}

In HORIZON-PFT, patients treated with zoledronic acid had an absolute increase of approximately $0.69 \%$ (3.34\% with zoledronic acid versus $2.65 \%$ with placebo) in inflammatory ocular adverse events, primarily conjunctivitis, during the first 15 days after infusion. ${ }^{1}$ In HORIZON-RFT, four patients in the zoledronic acid group $(0.4 \%)$ and one patient in the placebo group had an ocular event that was considered possibly or probably related to study drug according to an expert review. ${ }^{2}$

\section{Cardiovascular events}

In HORIZON-PFT, there were more cardiac rhythm disturbances in the group receiving zoledronic acid (266 patients, or 6.9\%) than placebo (203 patients, or 5.3\%, $P=0.003$ ) and serious atrial fibrillation, a subcategory of all arrhythmias, was more common with patients in the zoledronic acid group. ${ }^{1}$ A total of 50 patients in the zoledronic acid group had serious atrial fibrillation (1.3\%) compared with 20 patients $(0.5 \%)$ in the placebo group $(P<0.001)$. After adjudication, the number of patients whose atrial fibrillation was reported as a serious adverse event remained similar (50 in the zoledronic acid group and 17 in the placebo group). In most zoledronic acid recipients with atrial fibrillation (47/50), the event occurred more than 30 days after infusion. In a preplanned substudy of 559 patients who underwent electrocardiography, the prevalence of atrial fibrillation ( $2.1 \%$ in the zoledronic acid group and $2.8 \%$ in the placebo group) and other electrocardiographic abnormalities was not significantly different between the study groups. There was no significant difference in incidence of atrial fibrillation overall, and no difference in strokes, deaths due to strokes, myocardial infarction, or death from cardiovascular diseases. In HORIZON-RFT, ${ }^{2}$ there was no significant difference in cardiovascular events in the two groups and no difference in atrial fibrillation as a serious adverse event.

The clinical significance, if any, of the finding of increased risk of serious atrial fibrillation in patients treated with zoledronic acid in HORIZON-PFT is unclear. Cardiac arrhythmias have not been reported in other RCTs of patients treated with bisphosphonates, and retrospective studies have shown conflicting results, with some suggesting an increased risk $^{43}$ and others suggesting no increase in risk. ${ }^{44}$

\section{Mortality}

Patients treated with zoledronic acid in HORIZON-RFT had a $28 \%$ lower risk of all-cause mortality than those receiving placebo $(9.6 \%$ versus $13.3 \%, P=0.01) .{ }^{2}$ Factors responsible for the mortality reduction are not clear. The adjudication committee determined that in the zoledronic acid group 11 deaths $(1.0 \%)$ were from cardiovascular disease and seven $(0.7 \%)$ were from cerebrovascular disease; in the placebo group, there were 18 deaths $(1.7 \%)$ from cardiovascular disease and seven $(0.7 \%)$ from cerebrovascular disease. While osteoporotic fractures of the hip and spine are associated with increased mortality, ${ }^{44}$ and it seems intuitive that reduction in fracture risk would reduce mortality, this is the first time that a mortality benefit has been reported in a RCT with a drug for the treatment of osteoporosis. 


\section{Economic evidence}

The cost of zoledronic acid once yearly for the treatment of osteoporosis is similar to the annualized cost for monthly oral risedronate and ibandronate; as a result, it is expected to demonstrate comparable cost-effectiveness. Alendronate is the only generic bisphosphonate currently available. The WHO recently released a clinical tool for estimation of the 10-year probability of fracture, FRAX ${ }^{\mathrm{TM}} .{ }^{8}$ This can be used in combination with country-specific data such as fracture rates, healthcare costs associated with fractures, and societal willingness to pay, to develop cost-effective intervention thresholds for pharmacologic therapy. The NOF has evaluated the WHO methodology and determined that it can be adapted for use in the US, ${ }^{46}$ and a cost-effectiveness analysis identified 10-year fracture probabilities at which it is costeffective to treat. ${ }^{47}$ The latter used a Markov cohort model of annual US age-specific incidence of clinical hip, spine, forearm, shoulder, rib, pelvis, and lower leg fracture, costs in 2005 \$US, and quality-adjusted life years (QALYs) to assess the cost-effectiveness of osteoporosis treatment, assuming \$US600/year drug cost for five years with a 35\% reduction in fracture risk and acceptable cost to society of up to \$US60000 per QALY gained. The conclusion was that it is cost-effective to treat when the 10 -year probability of hip fracture using the $\mathrm{WHO}$ algorithm is approximately $3 \%$ or more.

The updated NOF guide for the prevention and treatment of osteoporosis ${ }^{13}$ considered cost-effectiveness in establishing its recommendations to treat postmenopausal women and men aged 50 years and older under the following circumstances: a hip or vertebral (clinical or morphometric) fracture; T-score -2.5 or below at the femoral neck, total hip or spine after appropriate evaluation to exclude secondary causes; low bone mass (T-score between -1.0 and -2.5 at the femoral neck, total hip, or spine) and 10-year probability of hip fracture $3 \%$ or greater or a 10 -year probability of major osteoporosis-related fracture $20 \%$ or greater based on the US-adapted WHO algorithm. The NOF lists zoledronic acid as an FDA-approved drug for the treatment of osteoporosis but does not categorize the cost-effectiveness of individual drugs and recognizes that clinical decisions on whom to treat and how to treat must consider factors in addition to cost-effectiveness.

\section{Resource utilization}

The US Surgeon General ${ }^{11}$ and the $\mathrm{NOF}^{13}$ have described osteoporosis as a public health problem of huge magnitude, with clinical consequences and healthcare costs that are expected to increase with the aging of the population. This is likely to extract a large toll in terms of personal suffering, lost productivity for the patient and family caregivers, and higher healthcare costs for individuals and society. The morbidity of osteoporotic fractures, especially those of the hip and spine, are a strain on interpersonal relationships between patients and their families. Osteoporosis-related fractures create a heavy economic burden, causing over 432000 hospital admissions, almost 2.5 million medical office visits, and about 180000 nursing home admissions annually in the US. ${ }^{11}$ The cost to the healthcare system associated with osteoporosis-related fractures has been estimated at \$US17 billion in 2005; hip fractures account for $14 \%$ of incident fractures and $72 \%$ of fracture costs. ${ }^{9}$ Due to the aging population, the Surgeon General estimates that the number of hip fractures and their associated costs could double or triple by the year 2040 .

The use of intravenous zoledronic acid $5 \mathrm{mg}$ every 12 months in appropriately selected patients can be expected to substantially reduce the risk of fractures. This in turn will result in fewer fracture-related hospitalizations, office visits, and nursing home admissions, as well as a reduction in fracture-related healthcare expenses.

\section{Patient group/population}

RCTs have shown fracture risk reduction with annual intravenous zoledronic acid $5 \mathrm{mg}$ in women with postmenopausal osteoporosis and in men and women with recent low-trauma hip fracture. The safety and tolerability profile has been generally very good. These groups can expect to benefit from fracture risk reduction with greater convenience and perhaps improved adherence compared with the more frequent oral dosing of other medications with proven fracture risk reduction. Zoledronic acid is an attractive option for women with postmenopausal osteoporosis because of the assured 12-month compliance, but can also be used in women who are not able to take oral bisphosphonates due to gastrointestinal intolerance, gastrointestinal contraindication, or malabsorption.

\section{Dosage, administration, and formulations}

Zoledronic acid is an injectable aminobisphosphonate with potent antiresorptive activity and high affinity for mineralizing bone. It is administered as a $5 \mathrm{mg}$ intravenous infusion through vented intravenous tubing over no less than $15 \mathrm{~min}$. It is supplied in a prepackaged bottle of zoledronic acid $5 \mathrm{mg} / 100 \mathrm{~mL}$. After opening the solution, it is stable for 
24 hours at $2-8^{\circ} \mathrm{C}\left(36-46^{\circ} \mathrm{F}\right)$. If refrigerated, the refrigerated solution should be allowed to reach room temperature before administration. Storage is recommended at $25^{\circ} \mathrm{C}\left(77^{\circ} \mathrm{F}\right)$ with excursions permitted to $15-30^{\circ} \mathrm{C}\left(59-86^{\circ} \mathrm{F}\right)$.

Patients, especially those on diuretic therapy, should be hydrated prior to administration of zoledronic acid; drinking several glasses of water before leaving home is probably sufficient for most patients. Zoledronic acid should be used with caution in patients taking other drugs that are potentially nephrotoxic. Since transient increases in serum creatinine may be greater in patients with impaired renal function, zoledronic acid is not recommended in patients with creatinine clearance less than $35 \mathrm{~mL} / \mathrm{min}$.

To reduce the risk of hypocalcemia and for general bone health, patients should have an adequate intake of calcium and vitamin D. The NOF recommends a daily intake of at least $1200 \mathrm{mg}$ elemental calcium with diet plus supplements, if needed, and vitamin $\mathrm{D}_{3} 800-1000 \mathrm{IU} /$ day.

\section{Clinical value}

Osteoporosis is a major public health problem associated with significant morbidity and mortality, resulting in a substantial personal burden to patients and caregivers, and an economic burden to individuals and a healthcare system with limited resources. It is underdiagnosed and undertreated, and when treated, many patients do not take medication correctly or do not take it long enough to achieve the expected benefit. Efforts to optimize skeletal health in all patients include regular physical activity, adequate intake of calcium and vitamin $\mathrm{D}$, and avoidance of cigarette smoking and excess alcohol. When drug therapy to reduce fracture risk is started, it is imperative that patients take medication regularly, correctly, and long enough to benefit from the reduction in fracture risk.

The use of once yearly intravenous zoledronic acid offers the potential to improve effectiveness in clinical practice by providing $100 \%$ bioavailability, eliminating concern of malabsorption of oral bisphosphonates due to intestinal disease or incorrect administration, and providing a drug that reduces bone turnover for at least one year after a single infusion. It has proven antifracture efficacy at all important skeletal sites in women with postmenopausal osteoporosis, and has been shown to reduce the risk of new clinical fractures, as well as all-cause mortality, in men and women with a previous hip fracture. It is associated with a favorable safety profile and was generally well tolerated in both large fracture prevention trials. Postdose, flu-like symptoms in clinical trials were transient, and in clinical practice such symptoms may be mitigated by treatment with acetaminophen or ibuprofen. Intravenous zoledronic acid is especially attractive for patients with gastrointestinal contraindications to oral bisphosphonates, gastrointestinal intolerance to oral bisphosphonates, or malabsorption of oral bisphosphonates.

In conclusion, zoledronic acid is an important new therapeutic option in the management of patients with osteoporosis, potentially leading to improved clinical outcomes and reducing the burden of osteoporotic fractures.

\section{Acknowledgments}

The author declares that he has received grant/research support from Merck, Eli Lilly, Novartis, sanofi aventis, Amgen, Pfizer, Wyeth, Roche, GSK, Procter \& Gamble; acted as a consultant and attended advisory boards, speakers' bureau, or sponsored speaking events with Merck, Eli Lilly, Novartis, Procter \& Gamble, sanofi aventis, Roche, GSK, Wyeth, Servier, Amgen, Upsher-Smith; and is a direct stock shareholder of General Electric, and Procter and Gamble.

\section{References}

1. Black DM, Delmas PD, Eastell R, et al. Once-yearly zoledronic acid for treatment of postmenopausal osteoporosis. $N$ Engl J Med. 2007;356:1809-1822.

2. Lyles KW, Colon-Emeric CS, Magaziner JS, et al. Zoledronic acid and clinical fractures and mortality after hip fracture. $N$ Engl J Med. 2007;357:1799-1809.

3. McClung M, Recker R, Miller R, et al. Intravenous zoledronic acid $5 \mathrm{mg}$ in the treatment of postmenopausal women with low bone density previously treated with alendronate. Bone. 2007;41:122-128.

4. Klibanski A, Adams-Campbell L, Bassford T, et al. Osteoporosis prevention, diagnosis, and therapy. JAMA. 2001;285:785-795.

5. WHO (World Health Organization). Assessment of fracture risk and its application to screening for postmenopausal osteoporosis. Geneva, Switzerland: WHO. 1994

6. NOF (National Osteoporosis Foundation). Fast facts on osteoporosis. National Osteoporosis Foundation 2008a. Available at: http://www. nof.org/osteoporosis/diseasefacts.htm (accessed August 25, 2008).

7. Reginster JY, Burlet N. Osteoporosis: a still increasing prevalence. Bone. 2006;38(2 Suppl 1):S4-S9.

8. Kanis JA, Johnell O. Requirements for DXA for the management of osteoporosis in Europe. Osteoporos Int. 2005;16:229-238.

9. Burge R, Dawson-Hughes B, Solomon DH, Wong JB, King A, Tosteson A. Incidence and economic burden of osteoporosis-related fractures in the United States, 2005-2025. J Bone Miner Res. 2007;22:465-475.

10. Brenneman SK, Barrett-Connor E, Sajjan S, Markson LE, Siris ES. Impact of recent fracture on health-related quality of life in postmenopausal women. J Bone Miner Res. 2006;21:809-816.

11. USDHHS (US Department of Health and Human Services). Bone health and osteoporosis: a report of the surgeon general. Rockville, MD: US Department of Health and Human Services, Office of the Surgeon General. 2004. Available at: http://www.surgeongeneral.gov/library/ bonehealth/Executive_Summary.html.

12. Gabriel SE, Tosteson AN, Leibson CL, et al. Direct medical costs attributable to osteoporotic fractures. Osteoporos Int. 2002;13:323-330.

13. NOF (National Osteoporosis Foundation). Clinician's Guide to Prevention and Treatment of Osteoporosis. Washington, DC: National Osteoporosis Foundation; 2008b. 
14. Kanis JA, on behalf of the World Health Organization Scientific Group. Assessment of osteoporosis at the primary health-care level. Technical Report. World Health Organization Collaborating Centre for Metabolic Bone Diseases, University of Sheffield, UK: Printed by the University of Sheffield; 2007.

15. KarpfDB, Shapiro DR, Seeman E, et al. Prevention of nonvertebral fractures by alendronate - a meta-analysis. JAMA. 1997;277:1159-1164.

16. Harris ST, Watts NB, Genant HK, et al. Effects of risedronate treatment on vertebral and nonvertebral fractures in women with postmenopausal osteoporosis - a randomized controlled trial. JAMA. 1999;282: 1344-1352.

17. Black DM, Thompson DE, Bauer DC, et al. Fracture risk reduction with alendronate in women with osteoporosis: the Fracture Intervention Trial. J Clin Endocrinol Metab. 2000;85:4118-4124.

18. McClung MR, Geusens R Miller PD, et al. Effect of risedronate on the risk of hip fracture in elderly women. $N$ Engl J Med. 2001;344: 333-340.

19. Neer RM, Arnaud CD, Zanchetta JR, et al. Effect of parathyroid hormone (1-34) on fractures and bone mineral density in postmenopausal women with osteoporosis. N Engl J Med. 2001;344:1434-1441.

20. Wysowski DK, Chang JT. Alendronate and risedronate: reports of severe bone, joint, and muscle pain. Arch Intern Med. 2005;165:346-347.

21. Khosla S, Burr D, Cauley J, et al. Bisphosphonate-associated osteonecrosis of the jaw: report of a task force of the American Society for Bone and Mineral Research. J Bone Miner Res. 2007;22:1479-1489.

22. Ettinger B, Black DM, Mitlak BH, et al. Reduction of vertebral fracture risk in postmenopausal women with osteoporosis treated with raloxifene - results from a 3-year randomized clinical trial. JAMA. 1999, 282:637-645.

23. Nelson HD, Humphrey LL, Nygren P, Teutsch SM, Allan JD. Postmenopausal hormone replacement therapy: scientific review. JAMA. 2002;288:872-881.

24. MacLennan A, Lester S, Moore V. Oral estrogen replacement therapy versus placebo for hot flushes: a systematic review. Climacteric. 2001;4:58-74.

25. Vahle JL, Sato M, Long GG, et al. Skeletal changes in rats given daily subcutaneous injections of recombinant human parathyroid hormone (1-34) for 2 years and relevance to human safety. Toxicologic Pathology. 2002;30:312-321.

26. Vahle JL, Long GG, Sandusky G, Westmore M, Ma YL, Sato M. Bone neoplasms in F344 rats given teriparatide [rhPTH(1-34)] are dependent on duration of treatment and dose. Toxicol Pathol. 2004;32:426-438.

27. Tashjian AH Jr, Gagel RF. Teriparatide [human PTH(1-34)]: 2.5 years of experience on the use and safety of the drug for the treatment of osteoporosis. J Bone Miner Res. 2006;21:354-365.

28. Nguyen TV, Center JR, Eisman JA. Osteoporosis: underrated, underdiagnosed and undertreated. Med J Aust. 2004;180(5 Supp1):S18-S22.

29. Siris ES, Harris ST, Rosen CJ, et al. Adherence to bisphosphonate therapy and fracture rates in osteoporotic women: relationship to vertebral and nonvertebral fractures from two US claims databases. Mayo Clin Proc. 2006;81:1013-1022.

30. Delmas PD, van de Langerijt L, Watts NB, et al. Underdiagnosis of vertebral fractures is a worldwide problem: the IMPACT study. J Bone Miner Res. 2005;20:557-563.

Core Evidence

\section{Publish your work in this journal}

Core Evidence is an international, peer-reviewed open-access journal evaluating the evidence underlying the potential place in therapy of drugs throughout their development lifecycle from preclinical to postlaunch. The focus of each review is to evaluate the case for a new drug or class in outcome terms in specific indications and patient
31. Vestergaard P, Rejnmark L, Mosekilde L. Osteoporosis is markedly underdiagnosed: a nationwide study from Denmark. Osteoporos Int. 2005;16:134-141.

32. Kamel HK. Secondary prevention of hip fractures among the hospitalized elderly: are we doing enough? J Clin Rheumatol. 2005;11:68-71.

33. Cramer JA, Amonkar MM, Hebborn A, Altman R. Compliance and persistence with bisphosphonate dosing regimens among women with postmenopausal osteoporosis. Curr Med Res Opin. 2005;21:1453-1460.

34. Recker RR, Gallagher R, MacCosbe PE. Effect of dosing frequency on bisphosphonate medication adherence in a large longitudinal cohort of women. Mayo Clin Proc. 2005;80:856-861.

35. Reginster JY, Rabenda V, NeuprezA. Adherence, patient preference and dosing frequency: understanding the relationship. Bone. 2006; 38(4 Suppl 1):S2-S6.

36. Cooper A, Drake J, Brankin E. Treatment persistence with once-monthly ibandronate and patient support vs once-weekly alendronate: results from the PERSIST study. Int J Clin Pract. 2006;60:896-905.

37. McCombs JS, Thiebaud R, Laughlin-Miley C, Shi J. Compliance with drug therapies for the treatment and prevention of osteoporosis. Maturitas. 2004;48:271-287.

38. Wasnich RD, Miller PD. Antifracture efficacy of antiresorptive agents are related to changes in bone density. J Clin Endocrinol Metab. 2000;85:231-236.

39. Hewitt RE, Lissina A, Green AE, Slay ES, Price DA, Sewell AK. The bisphosphonate acute phase response: rapid and copious production of proinflammatory cytokines by peripheral blood gd $\mathrm{T}$ cells in response to aminobisphosphonates is inhibited by statins. Clin Exp Immunol. 2005;139:101-111.

40. Woo SB, Hellstein JW, Kalmar JR. Narrative [corrected] review: bisphosphonates and osteonecrosis of the jaws. Ann Intern Med. 2006;144:753-761.

41. Grbic JT, Landesberg R, Lin SQ, et al. Incidence of osteonecrosis of the jaw in women with postmenopausal osteoporosis in the health outcomes and reduced incidence with zoledronic acid once yearly pivotal fracture trial. J Am Dent Assoc. 2008;139:32-40.

42. Lewiecki EM, Miller PD. Renal safety of intravenous bisphosphonates in the treatment of osteoporosis. Expert Opin Drug Saf. 2007;6:663-672.

43. Heckbert SR, Li G, Cummings SR, Smith NL, Psaty BM. Use of alendronate and risk of incident atrial fibrillation in women. Arch Intern Med. 2008;168:826-831.

44. Sorensen HT, Christensen S, Mehnert F, et al. Use of bisphosphonates among women and risk of atrial fibrillation and flutter: population based case-control study. BMJ. 2008;336:813-816.

45. Cauley JA, Thompson DE, Ensrud KC, Scott JC, Black D. Risk of mortality following clinical fractures. Osteoporos Int. 2000;11:556-561.

46. Dawson-Hughes B, Tosteson AN, Melton LJ III, et al. Implications of absolute fracture risk assessment for osteoporosis practice guidelines in the USA. Osteoporos Int. 2008;19:449-458.

47. Tosteson AN, Melton LJ III, Dawson-Hughes B, et al. Cost-effective osteoporosis treatment thresholds: the United States perspective. Osteoporos Int.2008;19:437-47. groups. The manuscript management system is completely online and includes a very quick and fair peer-review system, which is all easy to use. Visit http://www.dovepress.com/testimonials.php to read real quotes from published authors. 